\title{
Influência de diferentes meios de cultura sobre o crescimento de Pfaffia glomerata (Spreng.) Pedersen (Amaranthaceae) para conservação in vitro
}

\author{
ALVES, R.B.N. ${ }^{1}$; BERTONI, B.W. ${ }^{2}$; VIEIRA, R.F. ${ }^{1}$; FRANÇA, S.C. ${ }^{2}$; MING, L.C. ${ }^{3}$; PEREIRA, AM.S. ${ }^{3 *}$ \\ ${ }^{1}$ Embrapa Recursos Genéticos e Biotecnologia, PqEB W-5 Norte Final, CEP: 70770-900, Brasília-Brasil ${ }^{2}$ Universidade \\ de Ribeirão Preto, Avenida Costábile Romano, 2201, CEP: 14096-900, Ribeirão Preto-Brasil \\ *apereira@unaerp.br ${ }^{3}$ Departamento de Horticultura, FCA, Unesp, Caixa Postal 237, CEP: 18603-970, Botucatu-Brasil
}

\begin{abstract}
RESUMO: Pfaffia glomerata ocorre em vários estados do Brasil e países limítrofes da região Sul às margens de rios e nas orlas das matas de galerias, é espécie hidrófita e heliófita. As raízes de espécies do gênero Pfaffia são usadas na medicina popular brasileira, especialmente como tônico, afrodisíaco e no controle do diabete. O objetivo deste trabalho foi estabelecer um banco de germoplasma in vitro de Pfaffia glomerata. O experimento em delineamento inteiramente casualizado foi conduzido com seis tratamentos: 1 ) MS $+2 \%$ de sacarose $+4 \%$ de sorbitol; 2 ) $\mathrm{MS} / 2+2 \%$ de sacarose $+4 \%$ de sorbitol; 3 ) MS $+2 \%$ de sacarose $+4 \%$ de sorbitol $+2 \mathrm{mg} \mathrm{L}^{-1}$ de pantotenato de cálcio; 4) MS/2 $+2 \%$ de sacarose $+4 \%$ de sorbitol $+2 \mathrm{mg} \mathrm{L}^{-1}$ de pantotenato de cálcio; 5$) \mathrm{MS}+2 \%$ de sacarose $+3 \%$ de manitol $+2 \mathrm{mg} \mathrm{L}^{-1}$ de pantotenato de cálcio; 6 ) MS/ $2+2 \%$ de sacarose $+3 \%$ de manitol $+2 \mathrm{mg} \mathrm{L}^{-1}$ de pantotenato de cálcio. Os resultados obtidos foram submetidos à análise de variância e ao teste de separação de médias de Scott Knott. Os tratamentos um, três e quatro apresentaram, significativamente, o maior número de segmentos nodais por haste, quando comparados com os tratamentos dois, cinco e seis. O tratamento dois foi o mais indicado para a conservação in vitro da espécie por ter promovido menor crescimento das plantas (altura de 3,1 $\pm 1,9 \mathrm{~cm}$ ), alto índice de sobrevivência, 100\% de explantes com brotação e o maior número de brotos por explante, após seis meses de cultivo. Todas as plântulas produziram raízes e não houve formação de calos, também não ocorreu hiperhidricidade nos tratamentos avaliados. As plantas aclimatizadas apresentaram $100 \%$ de sobrevivência no ambiente ex vitro. A manutenção de acessos de $P$. glomerata no banco de germoplasma in vitro é viável tanto do ponto de vista da conservação quanto economicamente.
\end{abstract}

Palavras-chave: ginseng-brasileiro, conservação de recursos genéticos, banco de germoplasma

\begin{abstract}
Influence of different culture media on the growth of Pfaffia glomerata (Spreng.) Pendersen (Amaranthaceae) for conservation in vitro. Pfaffia glomerata occurs in several states of Brazil and its neighboring countries in the south region at riverbanks and gallery forests. It is a hydrophyte and heliophyte species. The roots of the genus Pfaffia are used in Brazilian folk medicine especially as tonic, aphrodisiac and to control diabetes. The aim of this work was to establish an in vitro germplasm bank for Pfaffia glomerata. The experiment was carried out in completely randomized design with six treatments: 1) $\mathrm{DM}+2 \%$ sucrose $+4 \%$ sorbitol; 2) $\mathrm{DM} / 2+2 \%$ sucrose $+4 \%$ sorbitol; 3$) \mathrm{DM}+2 \%$ sucrose $+4 \%$ sorbitol $+2 \mathrm{mg} \mathrm{L}^{-1}$ calcium pantothenate; 4$) \mathrm{DM} / 2+2 \%$ sucrose $+4 \%$ sorbitol $+2 \mathrm{mg} \mathrm{L}^{-1}$ calcium pantothenate; 5 ) $\mathrm{DM}+2 \%$ sucrose $+3 \%$ mannitol $+2 \mathrm{mg} \mathrm{L}^{-1}$ calcium pantothenate; 6$) \mathrm{DM} / 2+2 \%$ sucrose $+3 \%$ mannitol $+2 \mathrm{mg} \mathrm{L}^{-1}$ calcium pantothenate. Results were subjected to analysis of variance and Scott Knott test for mean grouping. Treatments 1, 3 and 4 had a significantly larger number of nodal segments per stem, compared to Treatments 2, 5 and 6. Treatment 2 was the most appropriate for the in vitro conservation of this species since it led to the lowest growth (3.1 1.9 $\mathrm{cm}$ height), high survival rate, $100 \%$ explants with sprouting, and the largest number of sprouts per explant after six months of culture. All seedlings produced root and showed no formation of calluses or hyperhydricidity under the evaluated treatments. Acclimatized plants showed $100 \%$ survival in the ex vitro environment. Maintaining $P$. glomerata accessions in an in vitro germplasm bank is viable both economically and for conservation.
\end{abstract}

Key words: Brazilian ginseng, conservation of genetic resources, germplasm bank

Recebido para publicação em 06/08/2008

Aceito para publicação em 22/09/2010

Rev. Bras. PI. Med., Botucatu, v.12, n.4, p.510-515, 2010. 


\section{INTRODUÇÃO}

A conservação ex situ de recursos genéticos encontra nos bancos e coleções de germoplasma as estruturas físicas onde são armazenadas as amostras de material vivo, representativas de um indivíduo, ou de vários indivíduos, na forma de sementes, plantas ou partes da planta, tecidos ou células, para a manutenção da variabilidade genética visando a sua utilização (IBPGR, 1991).

Nos últimos anos, as técnicas de cultura in vitro para conservação em banco de germoplasma foram amplamente desenvolvidas e aplicadas em mais de mil espécies, muitas das quais são de regiões tropicais (Lata, 1991). Para as espécies ameaçadas de extinção e cuja conservação por sementes não é possível, este é um método considerado altamente promissor (Hoyt, 1992).

Os acessos mantidos in vitro são submetidos a ambientes físico-químicos que diminuem o metabolismo e reduzem o crescimento da planta, sem afetar a viabilidade, possibilitando intervalos maiores entre os subcultivos reduzindo-se assim a mão-deobra e o espaço necessários para a conservação. A diminuição da atividade metabólica pode ocorrer pela redução da intensidade de luz ou temperatura, acréscimo de retardadores de crescimento ao meio de cultura ou diminuição da concentração dos componentes salinos e orgânicos do meio (Withers \& Williams, 1998). Nestas condições, a conservação de germoplasma in vitro pode ser realizada a partir de mudanças no ambiente de cultivo o qual é diferente do tradicionalmente utilizado para promover o crescimento de células, tecidos e órgãos. Portanto, os meios de cultivos apropriados para a conservação de germoplasma possuem composição diferente dos demais a fim de atender o objetivo de manter os acessos nos bancos e coleções pelo maior tempo possível entre subcultivos, sem comprometer a sobrevivência e a integridade dos materiais.

No Brasil, uma das iniciativas pioneiras no que se refere à conservação de recursos genéticos de plantas medicinais em cultura de tecidos foi a criação, em 1987, do banco de germoplasma in vitro de plantas medicinais da Universidade de Ribeirão Preto (Unaerp). Em 2001, o banco foi ampliado visando à introdução in vitro de espécies medicinais da família Bignoniaceae com atividade antitumoral. Atualmente, o banco de germoplasma conserva também espécies medicinais nativas de outras famílias, como por exemplo, Amaranthaceae, Asteraceae e outras.

Pfaffia glomerata (Amaranthaceae) ocorre em vários estados do Brasil e países limítrofes da região Sul. É comum encontrá-la na beira de rios e nas orlas das matas de galerias, onde podem receber bastante luz, pois é uma espécie hidrófita e heliófita (Smith \& Downs, 1972). A planta é típica também de campos de inundação de rios e é frequente no cerrado e matas do estado de Mato Grosso do Sul (Pott \& Pott, 1994). A distribuição geográfica de $P$. glomerata é ampla, ocupando condições climáticas e edáficas diferentes, resultando em populações com variabilidade genética (Magalhães, 2000).

As raízes de espécies do gênero Pfaffia são usadas na medicina popular brasileira, especialmente como tônico, afrodisíaco e no controle do diabete. Shiobara et al. (1993), analisando a composição química das raízes de $P$. glomerata, detectaram a presença de substâncias com atividade adaptógena (característica farmacológica de promover aumento da resistência), como ácido glomérico (triterpenóide), ácido pfamérico (nortriterpenóide), ecdisterona, rubrosterona, ácido oleanólico e $\beta$-glicopiranosil oleanolato.

A partir dos vários estudos sobre as atividades farmacológicas dos compostos presentes nas raízes de $P$. glomerata e de outras espécies do gênero houve grande interesse comercial. Assim, intensificou-se a exploração predatória das populações naturais da espécie, justificando as pesquisas para viabilizar planos de manejo sustentável nas áreas de ocorrência, projetos visando a produção comercial e o estabelecimento de bancos e coleções de germoplasma, fundamentais para amenizar a pressão ecológica sobre a espécie (Montanari Junior et al., 1999).

A coleta de germoplasma de P. glomerata em populações naturais e a manutenção destes materiais são ações importantes visando à conservação da espécie. Por outro lado, é extremamente relevante a avaliação deste germoplasma mantido em bancos e coleções como estratégia fundamental para a utilização.

Dentro deste contexto o presente trabalho teve como objetivo estudar a influência de diferentes meios de cultura sobre o crescimento de Pfaffia glomerata (Spreng.) Pedersen visando estabelecer um banco de germoplasma in vitro para a espécie.

\section{MATERIAL E MÉTODO}

O experimento foi conduzido no laboratório de cultura de tecidos da Unidade de Biotecnologia Vegetal da Universidade de Ribeirão Preto, Unaerp, SP. As plantas matrizes de Pfaffia glomerata (Spreng.) Pedersen (Amaranthaceae), para fornecimento dos explantes, foram obtidas no Parque Nacional de llha Grande, PR, em 2001 e em Itatinga, SP, no ano de 2004. Exsicatas foram encaminhadas ao Herbário Friburgense (FCAB), RJ, para identificação taxonômica pelo Prof. Dr. Josafá Carlos Siqueira. As exsicatas foram depositadas no Herbário FCAB, RJ, números de registro $F C A B$ 6092; FCAB 6095 e FCAB 6098 e no Herbário CEN, DF, número 62.024.

Os segmentos nodais de aproximadamente $1,0 \mathrm{~cm}$ e sem folhas foram obtidos de plântulas de Pfaffia glomerata (ginseng-brasileiro) micropropagadas mantidas sob condição in vitro. Estes explantes foram 
introduzidos nos seguintes meios de cultura: $\mathrm{T} 1) \mathrm{MS}$ $+2 \%$ de sacarose $+4 \%$ de sorbitol; T2) MS/2 + $2 \%$ de sacarose $+4 \%$ de sorbitol; T3) $M S+2 \%$ de sacarose $+4 \%$ de sorbitol $+2 \mathrm{mg} \mathrm{L}^{-1}$ de pantotenato de cálcio; T4) $\mathrm{MS} / 2+2 \%$ de sacarose $+4 \%$ de sorbitol $+2 \mathrm{mg}$ $\mathrm{L}^{-1}$ de pantotenato de cálcio; T5) $\mathrm{MS}+2 \%$ de sacarose $+3 \%$ de manitol $+2 \mathrm{mg} \mathrm{L}^{-1}$ de pantotenato de cálcio; T6) $\mathrm{MS} / 2+2 \%$ de sacarose $+3 \%$ de manitol $+2 \mathrm{mg}$ $\mathrm{L}^{-1}$ de pantotenato de cálcio.

O delineamento experimental utilizado foi o inteiramente casualizado com 30 repetições por tratamento. A parcela experimental consistiu de cada frasco de vidro de $8 \mathrm{~cm}$ de altura $\times 5,5 \mathrm{~cm}$ de diâmetro (tipo cubeta) com tampa de plástico e um segmento nodal. O meio de cultivo foi o MS basal (Murashige e Skoog, 1962) solidificado com $0,3 \%$ de Phytagel $\circledast$, nas concentrações descritas para os tratamentos, com pH ajustado para 5.8, autoclavado a 1 atmosfera de pressão (atm), a $120^{\circ} \mathrm{C}$, por 15 minutos.

Os explantes foram inicialmente mantidos em temperatura de $16^{\circ} \mathrm{C}( \pm 1)$, umidade relativa de 60 $\pm 5 \%, 16 \mathrm{~h}$ de fotoperíodo e intensidade luminosa de $40 \mu \mathrm{mol} \mathrm{m}^{-2} \mathrm{~s}^{-1}$, fornecida por lâmpada fluorescente branca. Após seis meses, foi avaliado o índice de sobrevivência, o número de explantes com brotações, a altura das plântulas, o número de brotos por explante, o número de segmentos nodais por haste, a porcentagem de enraizamento, presença de calos e a ocorrência de hiperhidricidade. Os dados obtidos foram submetidos à análise de variância e ao teste de separação de médias de Scott Knott a $5 \%$ de probabilidade.

Após o cultivo por seis meses sob essas condições, as cubetas foram transferidas para sala de crescimento, com temperatura de $25^{\circ} \mathrm{C}$, permanecendo por cinco dias. Em seguida, as tampas das cubetas foram removidas e as plântulas permaneceram na sala de crescimento por mais cinco dias. Ao final deste período, as raízes foram lavadas com água corrente para retirada dos resíduos de meio de cultura e as plântulas semeadas em bandejas de poliestireno com 72 células, contendo uma mistura de vermiculita e Plantmax ${ }^{\circledR}(2: 1)$ como substrato. O plantio foi realizado após as $16 \mathrm{~h}$ para evitar transpiração excessiva e cada célula foi coberta com frascos de vidro. As bandejas foram transferidas para mini estufas de plástico, esse procedimento possibilitou a formação de um micro clima favorável ao estabelecimento das plântulas. Após cinco dias, as mini estufas foram retiradas e as bandejas foram levadas para casa de vegetação com temperatura média de $29^{\circ} \mathrm{C}$ e irrigação diária, por 10 dias. Posteriormente, as plantas foram transferidas para sacos de polietileno preto de $12 \mathrm{~cm}$ de largura $\times 22 \mathrm{~cm}$ de comprimento $\times 0,5 \mathrm{~cm}$ de espessura com solo, permanecendo na casa de vegetação por 40 dias, até o momento da avaliação da porcentagem de sobrevivência no ambiente ex vitro.

\section{RESULTADO E DISCUSSÃO}

Os segmentos nodais cultivados em MS + $2 \%$ de sacarose $+4 \%$ de sorbitol ( $\mathrm{T} 1)$ apresentaram $97 \%$ de sobrevivência e desenvolveram-se significativamente mais quando comparados com os segmentos mantidos em T2, provavelmente, pela maior disponibilidade de nutrientes. Aúnica diferença na composição do meio entre os tratamentos $\mathrm{T} 1 \mathrm{e}$ T2 foi a concentração dos mesmos (MS e M/2, respectivamente). No tratamento T2 o crescimento dos explantes foi de $3,1 \mathrm{~cm}$, ocorreu maior número de brotos por explante e alto índice de sobrevivência $(100 \%)$ após seis meses de cultivo (Tabela 1). Estes

TABELA 1. Índice de sobrevivência (\%), explantes com brotação (\%), altura da plântula (cm), número de brotos por explante, número de segmentos nodais por haste, enraizamento e formação de calos, em seis tratamentos para definição do meio de cultura no estabelecimento do banco de germoplasma de Pfaffia glomerata. Ribeirão Preto, SP, 2007.

\begin{tabular}{cccccccc}
\hline Tratamentos & $\begin{array}{c}\text { Índice de } \\
\text { sobrevivência } \\
(\%)\end{array}$ & $\begin{array}{c}\text { Explantes } \mathrm{C} / \\
\text { brotação }(\%)\end{array}$ & Altura $(\mathrm{cm})$ & $\begin{array}{c}\mathrm{N} \text { - de brotos/ } \\
\text { explante. }\end{array}$ & $\begin{array}{c}\mathrm{N} \text { - de segmentos } \\
\text { nodais/haste }\end{array}$ & $\begin{array}{c}\text { Enraizamento Formação de } \\
(\%)\end{array}$ & calos \\
\hline 1 & $97 \mathrm{a}$ & $96 \mathrm{a}$ & $4,6 \mathrm{a}$ & $1,8 \mathrm{a}$ & $5,4 \mathrm{a}$ & $100 \mathrm{a}$ & ausência \\
2 & $100 \mathrm{a}$ & $100 \mathrm{a}$ & $3,1 \mathrm{~b}$ & $1,9 \mathrm{a}$ & $4,3 \mathrm{~b}$ & $100 \mathrm{a}$ & ausência \\
3 & $100 \mathrm{a}$ & $100 \mathrm{a}$ & $4,5 \mathrm{a}$ & $1,7 \mathrm{a}$ & $5,6 \mathrm{a}$ & $100 \mathrm{a}$ & ausência \\
4 & $100 \mathrm{a}$ & $100 \mathrm{a}$ & $5,3 \mathrm{a}$ & $1,8 \mathrm{a}$ & $5,9 \mathrm{a}$ & $100 \mathrm{a}$ & ausência \\
5 & $83 \mathrm{~b}$ & $83 \mathrm{~b}$ & $0,2 \mathrm{c}$ & $1,7 \mathrm{a}$ & $1,4 \mathrm{c}$ & $100 \mathrm{a}$ & ausência \\
6 & $57 \mathrm{c}$ & $57 \mathrm{c}$ & $0,1 \mathrm{c}$ & $1,2 \mathrm{~b}$ & $0,9 \mathrm{c}$ & $100 \mathrm{a}$ & ausência \\
\hline
\end{tabular}

Médias seguidas pela mesma letra não diferem significativamente, entre si, ao nível de 1\% pelo teste de Scott Knott. T1: MS + 2\% de sacarose $+4 \%$ de sorbitol; T2: MS $/ 2+2 \%$ de sacarose $+4 \%$ de sorbitol; T3: MS $+2 \%$ de sacarose $+4 \%$ de sorbitol +2 mg L-1 de pantotenato de cálcio; T4: $\mathrm{MS} / 2+2 \%$ de sacarose $+4 \%$ de sorbitol $+2 \mathrm{mg} \mathrm{L}{ }^{-1}$ de pantotenato de cálcio; T5: MS + $2 \%$ de sacarose $+3 \%$ de manitol $+2 \mathrm{mg} \mathrm{L}^{-1}$ de pantotenato de cálcio; T6: MS/2 + $2 \%$ de sacarose + 3\% de manitol + $2 \mathrm{mg} \mathrm{L}^{-1}$ de pantotenato de cálcio.

Rev. Bras. PI. Med., Botucatu, v.12, n.4, p.510-515, 2010. 
resultados são importantes para se viabilizar o aumento do intervalo de subcultivo. Desta forma, dentre os tratamentos avaliados o T2 foi o meio mais indicado para os propósitos da conservação in vitro de P. glomerata.

Os tratamentos (T3) e (T4), com sorbitol e suplementado com pantotenato de cálcio, possibilitaram índice de sobrevivência de $100 \%$ e o número de segmentos nodais por haste foi significativamente maior que o tratamento T2, T5 e T6. No entanto, a altura de brotação foi maior em relação aos outros tratamentos $(4,5 \mathrm{~cm}$ e $5,3 \mathrm{~cm}$, respectivamente) o que não é vantajoso para os propósitos da conservação in vitro já que os intervalos entre os subcultivos seriam menores.

Os explantes inoculados nos meios $\mathrm{MS}+2 \%$ de sacarose $+3 \%$ de manitol $+2 \mathrm{mg} \mathrm{L}^{-1}$ de pantotenato de cálcio (T5) e MS/2 $+2 \%$ de sacarose $+3 \%$ de manitol $+2 \mathrm{mg} \mathrm{L}^{-1}$ de pantotenato de cálcio (T6) apresentaram, estatisticamente, o menor número de segmentos nodais por haste $(1,4$ e 0,9$)$ e menor altura $(0,2$ e 0,1$)$ (Tabela 1). Os explantes mantidos nestes meios foram também os que tiveram menores índices de sobrevivência 83,3 \pm 4\% (T5) e 56,6 $\pm 5 \%$ (T6) o que constitui desvantagem para o uso destes meios de cultivos na conservação de germoplasma. $O$ crescimento das plântulas e os valores para todas as características avaliadas foram menores, em comparação com os demais tratamentos, portanto, é pouco provável que tenha ocorrido exaustão do meio. Desta forma, o manitol ou a combinação desse composto com pantotenato de cálcio parece ter exercido efeito prejudicial à manutenção das plântulas de $P$. glomerata. Quanto à porcentagem de explantes com brotações pode-se também concluir que a substituição do sorbitol pelo manitol interferiu negativamente na produção dos brotos quando estes foram mantidos sob temperatura de $16^{\circ} \mathrm{C}$ no banco de germoplasma in vitro. Lemos e Baker (1998) relataram efeitos nocivos do manitol em internós de Annona muricata cultivados in vitro. Bertoni et al. (2007) também verificaram menor índice de sobrevivência das plântulas de Zeyheria montana em presença de manitol. Fortes e Pereira (2001) avaliaram o efeito da sacarose, do manitol e do ácido acetilsalicílico (AAS) na preservação in vitro de batata (Solanum tuberosum L.), cultivar Macaca. O uso do manitol reduziu o crescimento das hastes e a formação de gemas nas brotações, o que não é desejável para espécies que se propagam vegetativamente. Os autores inferiram que as hastes de batata sofreram maior estresse fisiológico, devido principalmente, ao estresse osmótico provocado pelo manitol, que reduz a absorção de água e nutrientes.

Neste trabalho realizado com $P$. glomeratanão houve formação de calos nos explantes (Figura 1) nem sinais de hiperhidricidade, indicando que as concentrações dos componentes dos meios e do agente gelificante são adequadas para a cultura da espécie. Outro fator que pode ter contribuído para a ausência de hiperhidricidade foi à umidade relativa do ar (UR) da sala de crescimento em torno de $60 \%$, alguns trabalhos têm demonstrado que ambientes com elevado índice de umidade favorece a hiperhidricidade (Gribble, 1999; Moraes et al., 2007). Brotos que se desenvolvem em condições contínuas de alta umidade relativa apresentam maior susceptibilidade à hiperhidricidade, pois este é provavelmente o ambiente mais favorável para ocasioná-la (Guerra e Nodari, 2006).

Foi observado também que cada explante deu origem, em geral, a duas brotações sendo uma bem mais desenvolvida do que a outra. Este padrão de desenvolvimento também foi constatado em trabalhos realizados com segmentos nodais de Pfaffia tuberosa (Martins \& Nicoloso, 2004) e Pfaffia glomerata (Nicoloso et al., 2001) avaliados em meio de cultivo MS. Os autores verificaram também que cultivos subseqüentes por mais de dois anos, nas mesmas condições, mantiveram o padrão.

Nenhum tratamento inibiu a formação de raízes, ocorrendo $100 \%$ de enraizamento em explantes de $P$. glomeratanas condições do experimento (Tabela 1). As plântulas aclimatizadas apresentaram $100 \%$ de sobrevivência e após dois meses no ambiente ex vitro, foi observada produção de flores e sementes, este resultado pode ser considerado promissor para o cultivo comercial de $P$. glomerata produzida in vitro. Skrebsky et al. (2006) também utilizaram com sucesso um período de sete semanas na aclimatização de $P$. glomerata e o tempo de aclimatização foi considerado um fator econômico importante na produção de mudas visando à produção comercial em escala.

Desta forma, a manutenção de acessos de $P$. glomerata em banco de germoplasma in vitroé viável e eficiente, tanto do ponto de vista da conservação quanto economicamente. Assim, foram introduzidos no Banco de Germoplasma in vitro da Universidade de Ribeirão Preto (UNAERP) oitenta genótipos de $P$. glomerata. A variabilidade genética da espécie conservada em banco de germoplasma poderá contribuir para as pesquisas sobre vários aspectos da farmacologia, biologia reprodutiva e genética de populações, assim como compreender o processo evolutivo da espécie e seu papel ecológico. Além disso, a avaliação dos genótipos com objetivo de encontrar fontes de resistência às doenças também poderá viabilizar o plantio comercial.

\section{AGRADECIMENTO}

À Fapesp pelo apoio financeiro do projeto de pesquisa número 05/60751-3. À Unesp-Botucatu pelo apoio na coleta do material e à Unaerp pela disponibilização de laboratórios, casa de vegetação 


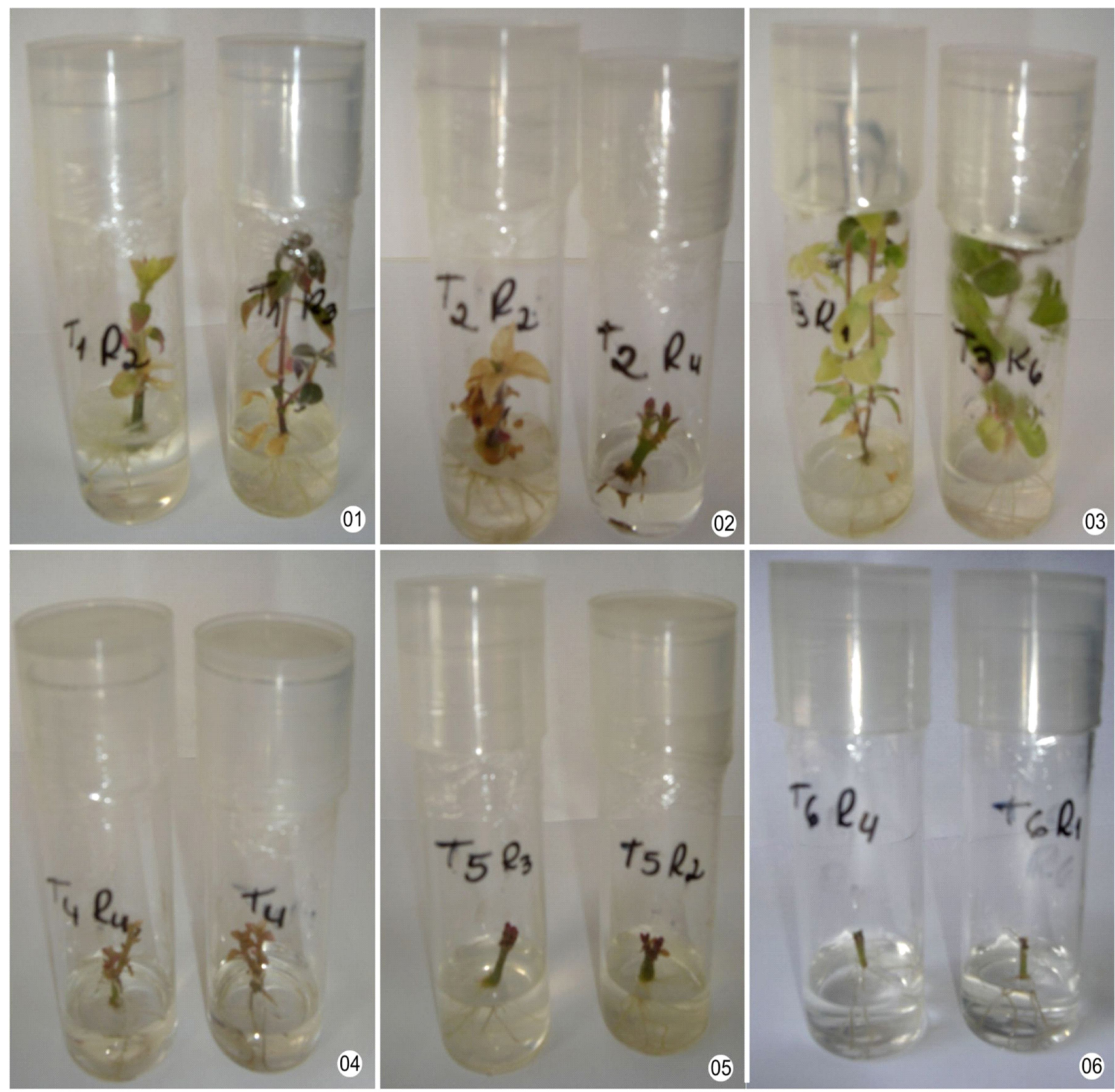

FIGURA 1. Plântulas de Pfaffia glomerata submetidas a seis tratamentos para definição do meio de cultura no estabelecimento de banco de germoplasma in vitro, após seis meses de cultivo, Ribeirão Preto, SP, 2006 (Foto: Paulo Vinícius A. de Oliveira). T1 $=M S+2 \%$ sacarose $+4 \%$ de sorbitol; T2 $=M S / 2+2 \%$ sacarose $+4 \%$ de sorbitol; T3 $=\mathrm{MS}+2 \%$ sacarose $+4 \%$ de sorbitol $+2 \mathrm{mg} \mathrm{L}^{-1}$ pantotenato de cálcio; $\mathrm{T} 4=\mathrm{MS} / 2+2 \%$ sacarose + $4 \%$ de sorbitol $+2 \mathrm{mg} \mathrm{L}^{-1}$ pantotenato de cálcio; $\mathrm{T} 5=\mathrm{MS}+2 \%$ sacarose $+3 \%$ de manitol $+2 \mathrm{mg} \mathrm{L}^{-1}$ pantotenato de cálcio; $\mathrm{T} 6=\mathrm{MS} / 2+2 \%$ sacarose $+3 \%$ de manitol $+2 \mathrm{mg} \mathrm{L}^{-1}$ pantotenato de cálcio.

e banco de germoplasma. À Embrapa pelo apoio institucional durante a realização do experimento.

\section{REFERÊNCIA}

BERTONI, B.W. et al. Zeyheria montana Mart. In: PEREIRA, A.M.S. (Org.). Recursos genéticos e conservação de plantas medicinais do Cerrado. Ribeirão Preto: Legis Summa, 2007. p.294-326.

FORTES, G.R.L.; PEREIRA, J.E.S. Preservação in vitro da batata com ácido acetilsalicílico e duas fontes de carboidrato. Pesquisa Agropecuária Brasileira, v.34, n.10, p.1261-4, 2001.
GRIBBLE, K. The influence of relative humidity on vitrification, growth and morphology of Gypsophila paniculata L. Plant Growth Regulation, v.27, p.179-88, 1999.

GUERRA, M.P.; NODARI, R.O. Apostila de Biotecnologia 1: cultura de tecidos vegetais. Florianópolis: UFSC/ Steinmacher. 2006. 41p.

HOYT, E. Conservação dos parentes silvestres de plantas cultivadas. Wilminton: Addison-Wesley Iberoamericana, 1992. 52p.

IBPGR. Report of the third external review of the International Board for Plant Genetics Resources. Rome: International Board for Plant Genetic Resources, 1991. 85p. 
LATA, A.H. In vitro conservation of tropical plant germoplasma, a review. Euphytica, v.57, p.227-43, 1991. LEMOS, E.E.P.; BAKER, D. Shoot regeneration in response to carbon source on internodal explants of Annona muricata L. Plant Growth Regulation, v.25, p.105-12, 1998.

MAGALHÃES, P.M. Agrotecnología para el cultivo de fáfia o "ginseng" brasilero. In: MARTíNEZ, J.V.A.; BERNAL, H.Y.; CÁCERES, A. (Orgs.). Fundamentos de agrotecnología de cultivo de plantas medicinales iberoamericanas. Santafé de Bogotá: Andrés Bello/CYTED, 2000. p.32332.

MARTINS, C.F.; NICOLOSO, F.T. Micropropagação de Pfaffia tuberosa (Spreng.) Hicken. Revista Brasileira de Plantas Medicinais, v.6, n.3, p53-61, 2004.

MONTANARI JUNIOR, I.; MAGALHÃES, P.M.; QUEIROGA, C.L. Influence of plantation density and cultivation cycle on root productivity and tenors of â-ecdysone in Pfaffia glomerata (Spreng) Pedersen. Acta Horticulturae, v.3, n.502, p.125-8, 1999.

MORAES, R.M. et al. Micropropagação e banco de germoplasma in vitro para produção e conservação de plantas nativas do Cerrado. In: PEREIRA, A.M.S. Recursos genéticos e conservação de plantas medicinais do Cerrado. Ribeirão Preto: Legis Summa,
2007. p.185-211.

MURASHIGE, T; SKOOG, F. A revised medium for rapid growth and bio assays with tobacco tissue cultures. Physiology Plantarum, v.15, p.473-97, 1962.

NICOLOSO, F.T. et al. Micropropagação do ginseng brasileiro [Pfaffia glomerata (Spreng.) Pedersen]. Revista Brasileira de Plantas Medicinais, v.3, n.2, p.11-8, 2001. POTT, A.; POTT, V.S. Plantas do pantanal. Corumbá: Embrapa-SPI, 1994. 320p.

SHIOBARA, Y. et al. A nortriterpenoid, triterpenoid and ecdysteroids from Pfaffia glomerata. Phytochemistry, v.36, p.1527-30, 1993.

SKREBSKY, E.C.; NICOLOSO, F.T.; MALDANER, J. Substratos na aclimatização de Pfaffia glomerata (Spreng.) Pedersen produzida in vitro sob diferentes doses de sacarose. Ciência Rural, v.36, n.5, p.1416-23, 2006.

SMITH, L.B.; DOWNS, R.J. Amaranthaceae de Santa Catarina. Flora llustrada Catarinense. Itajaí: Herbário Barbosa Rodrigues, 1972. p.35-50.

WITHERS, L.A.; WILLIAMS, J.T. Conservação in vitro de recursos genéticos de plantas. In: TORRES, A.C.; CALDAS, L.S.; BUSO, J.A. (Eds.). Cultura de tecidos e transformação genética de plantas. Brasília: Embrapa, 1998. v.1, p.297-330. 